\title{
Automatisierte Bestimmung von Dentalmaterialien aus Speichel
}

\author{
KERSTIN THUROW ${ }^{1}$, THOMAS RODDELKOPF ${ }^{2}$, MAX ROHDE ${ }^{3}$, JONE BARTEL ${ }^{3}$, \\ HEIDI FLEISCHER ${ }^{2}$ \\ ${ }^{1}$ CENTER FOR LIFE SCIENCE AUTOMATION, UNIVERSITÄT ROSTOCK \\ ${ }^{2}$ INSTITUT FÜR AUTOMATISIERUNGSTECHNIK, UNIVERSITÄT ROSTOCK \\ ${ }^{3}$ INNERSTÄDTISCHES GYMNASIUM ROSTOCK
}

\section{Due to their excellent properties, methacrylates are often used in} tooth restoration. However, numerous studies have shown the occurrence of allergies in both patients and medical personnel. Thus, a precise knowledge of the concentrations of dental components from tooth materials is required. An automated solid phase extraction method was developed, which was evaluated for the determination of MA, MMA and HEMA in aqueous solutions and artificial saliva. The method allows the detection of the compounds in the lower ppb range with coefficients of variation between 1.97 and 4.55 per cent.

DOI: $10.1007 / \mathrm{s} 12268-020-1347-9$

(C) Die Autoren 2020

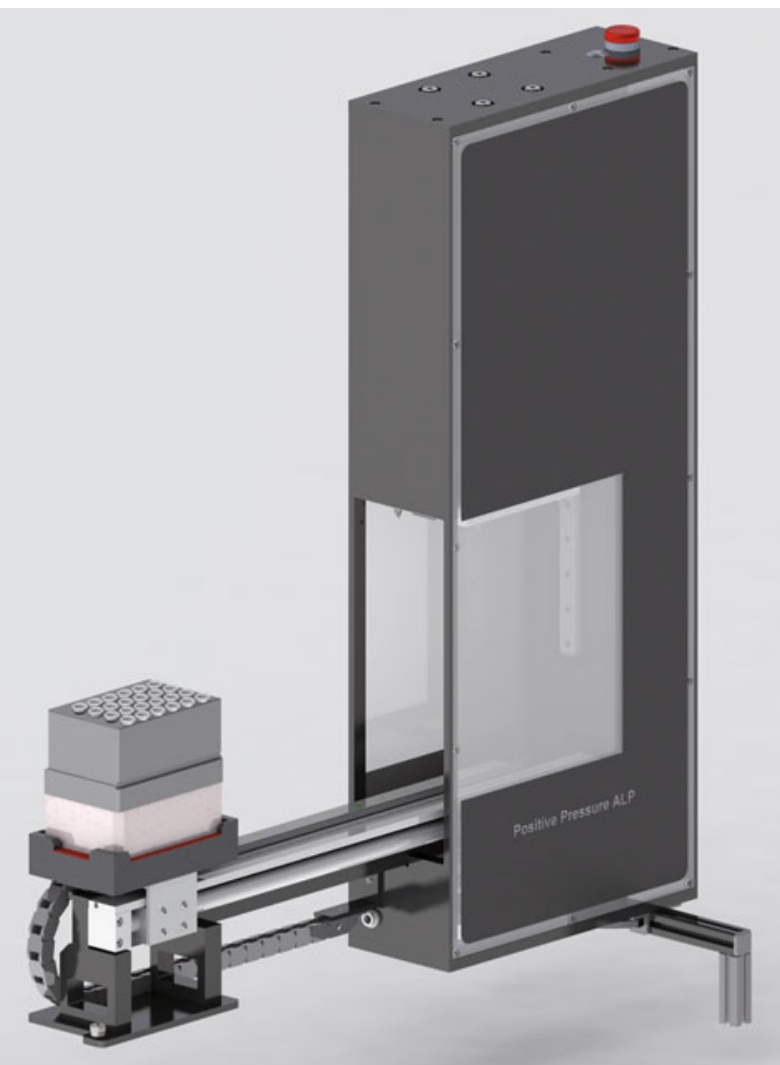

Methacrylate, z. B. Methacrylat (MA), Methylmethacrylat (MMA) und 2-Hydroxyethylmethacrylat (HEMA), finden in der restaurativen Zahnmedizin in Kompositmaterialien umfangreiche Anwendung, da durch Einbindung unterschiedlicher Zusatzstoffe, wie z. B. Metalloxid-Nanopartikel, Farbe und Transparenz dem natürlichen Zahn hervorragend angepasst werden können. Im Hinblick auf den Einsatz von Kompositmaterialien wurde in der zahnärztlichen Praxis vielfach über auftretende Kontaktallergien sowohl bei Patienten als auch bei zahnärztlichem Personal berichtet [1]. Dabei wurden allergische Reaktionen, Hypersensitivität, asthmatische Reaktionen, Irritationen der Haut sowie lokale neurobiologische Symptome beschrieben [2]. Aktuelle Untersuchungen zeigen, dass HEMA unter anderem genotoxische und cytotoxische Wirkungen zeigt $[3,4]$.

Während im Zuge einer Zahnrestauration geeignete Maßnahmen ergriffen werden können, um eine Exposition von Patienten und Personal mit den genannten Verbindungen zu reduzieren oder zu vermeiden, lässt sich eine spätere Elution von MMA durch Leaching- und Abbauprozesse (verursacht durch thermische und chemische Einflüsse sowie mechanische Einwirkungen durch Kaubewegungen) kaum vermeiden. Für die Ermittlung des potenziellen Risikos für Patienten, insbesondere bei bekannter Allergieneigung, müssen daher quantitative Angaben zur Konzentration von MMA und anderen Dentalwerkstoffen im Speichel gewonnen werden. Im Rahmen einer umfangreichen Untersuchung wurde daher ein geeignetes Verfahren für die automatisierte Bestimmung von MA, MMA und HEMA aus wässrigen Lösungen sowie künstlichem Speichel entwickelt.

Abb. 1: System für die automatisierte Festphasenextraktion (SPE). Der Stack mit Kartuschen und Zielgefäßen wird für die SPE in den Druckturm hineingefahren. 


\section{Methoden zur analysenmess- technischen Bestimmung von Methacrylaten}

Die analytische Bestimmung von MA, MMA und HEMA ist über massenspektrometrische Verfahren in Kopplung mit Gaschromatographie (GC) oder Hochleistungsflüssigkeitschromatographie (HPLC) möglich. W. Spahl et al. berichteten über Untersuchungen zum Auslaugverhalten von polymerisierten Zahnmaterialien [5]. Die Materialien wurden für drei Tage in Methanol oder Wasser eluiert. Die Eluate wurden direkt mittels GC/Massenspektrometrie (MS) bzw. Flüssigchromatographie(LC)/ MS analysiert. Eine weitere HPLC-basierte Messmethode [6] konnte durch H. Fleischer et al. bestätigt werden, wobei bei der Messpräzision (zehnfache Messung einer Probe) relative Standardabweichungen (Variationskoeffizienten) von maximal 2,8 Prozent (HPLC/MS) bzw. 1,4 Prozent (GC/MS) erzielt wurden [7].

Für die Bestimmung der Komponenten aus komplexeren Matrizes ist eine vorgelagerte Probenvorbereitung erforderlich. P. J. Babidge und W. J. Babidge beschrieben die Bestimmung von MMA aus Plasma bzw. Urin [8]. Die angesäuerten Proben wurden mit Ethylacetat extrahiert. Die Extrakte wurden getrocknet und mit Monodansylcadaverin und Dicyclohexylcarbodiimid derivatisiert. Für die Bestimmung mittels HPLC wurde Ethylmalonsäure als interner Standard eingesetzt. Die Nachweisgrenzen lagen bei $0,2 \mu \mathrm{mol} / \mathrm{l}$ MMA.

\section{Automatisierte Bestimmung von Methacrylaten mittels SPE}

Extraktionen mit organischen Lösungsmitteln sind sehr zeitaufwendig, wodurch die maximale Anzahl der pro Tag prozessierbaren Proben begrenzt wird. Eine gute Alternative stellt die Festphasenextraktion (solid phase extraction, SPE) dar. In Abhängigkeit von der zu bestimmenden Substanzklasse können unterschiedliche Sorbenzien eingesetzt werden.

Für die Untersuchungen wurden Stammlösungen der Methacrylate mit einer Konzentration von 10.000 ppm in Methanol angesetzt. Künstlicher Speichel (Neues Rezeptur-Formularium, NRF 7.5) wurde nach Garbe und Reimann hergestellt [9]. Die finale Konzentration der Methacrylate in den wässrigen Proben betrug $50 \mathrm{ppm}$.
Für die Untersuchungen wurde ein automatisiertes System für die Festphasenextraktion eingesetzt (celisca, Rostock) (Abb. 1). Für eine vollständige Automatisierung wurde in die SPE der Liquid-Handler Biomek NX S8 (Beckman Coulter, Krefeld) integriert, sodass alle Dosierschritte automatisiert durchgeführt werden können. Mittels eines Labware-Adapters wurden 24 Strata $^{\mathrm{TM}}-\mathrm{X}$-Kartuschen $(33 \mu \mathrm{m}$, polymeric reversed phase, $60 \mathrm{mg}$ ) über einer Mikrotiterplatte positioniert (Abb. 2). Zur Konditionierung wurden jeweils $900 \mu$ l Methanol und Wasser auf die Kartuschen gegeben und diese in die SPE-Einheit transportiert. Die Elution des Konditionierungsmittels erfolgte mit einem Druck von 0,35 bar für 15 Sekunden. Anschließend wurden zweimal $500 \mu \mathrm{l}$ Probe auf die SPE-Säulen aufgegeben und diese nach einer Wartezeit von 120 Sekunden für 90 Sekunden mit 0,5 bar Druck beaufschlagt. Die Kartuschen wurden zweimal mit je $750 \mu \mathrm{l}$ Methanol/Wasser (5:95; v/v) bei 0,35 bar für 75 bzw. 60 Sekunden gewaschen. Nach einer Trocknungszeit von 10 Minuten (1 bar) erfolgte die finale Elution der gewünschten Komponenten durch zweimalige Aufgabe von $500 \mu \mathrm{l}$ Dichlormethan und einer Elutionszeit von 60 Sekunden bei 0,35 bar. Die Extrakte wurden in Standard-GC-Vials eluiert. Zu Vergleichszwecken wurde eine manuelle, vakuumbasierte Festphasenextraktion durchgeführt.

Die quantitative Bestimmung von MA, MMA und HEMA erfolgte in Anlehnung an [8] auf einem 6890-Gaschromatographen mit einem massenselektivem 5973-Network-Detektor (beides Agilent Technologies, Santa Clara, USA) auf einer Chromatographiesäule HP5-MS (30 m) [10, 11].

Tabelle 1 zeigt eine Zusammenstellung der erhaltenen Ergebnisse zur Wiederholpräzision und Wiederfindungsraten für die Extraktion der Komponenten MA, MMA und HEMA aus Wasser und künstlichem Speichel. Dazu wurden jeweils 24 Proben (Wasser oder künstlicher Speichel) extrahiert und messtechnisch untersucht.

MA und HEMA zeigten in der automatisierten SPE deutlich höhere Wiederfindungsraten von 90,78-98,54\% bzw. 85,7$104,18 \%$ bei der Extraktion aus Wasser. Mittels manueller SPE wurden lediglich Wiederfindungsraten von 54,9-68,6\% (MA) sowie 55,18-67,42\% (HEMA) erhalten. Für die Extraktion aus Speichel ergibt
Hier steht eine Anzeige. Springer 


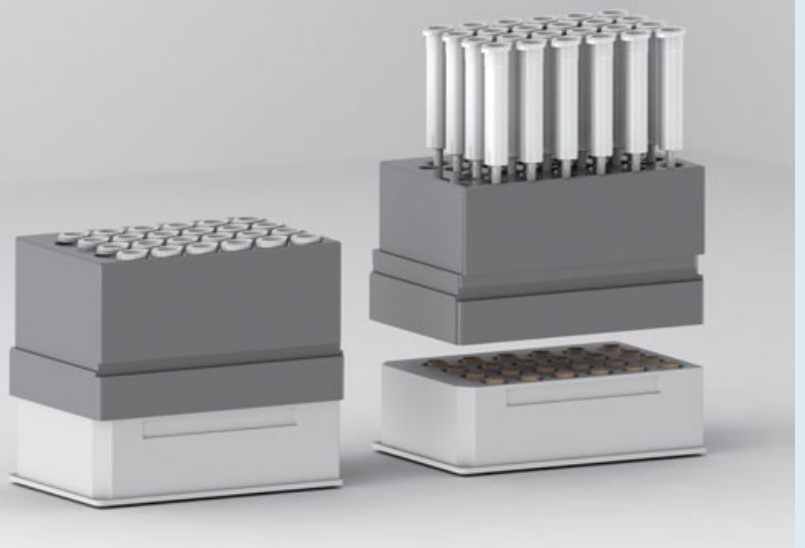

Tab. 1: Vergleich der Wiederfindungsraten und der Wiederholpräzision bei manueller und automatisierter Festphasenextraktion (SPE).

\begin{tabular}{lllll} 
& \multicolumn{2}{c}{ Manuelle SPE } & \multicolumn{2}{c}{ Automatisierte SPE } \\
\hline & Wasser & Speichel & Wasser & Speichel \\
\hline MA & & & & \\
\hline Max. Wiederfindungsrate [\%] & 68,6 & 56,64 & 98,54 & 92,96 \\
\hline Variationskoeffizient [\%] & 4,95 & 9,50 & 1,97 & 2,58 \\
\hline MMA & & & & \\
\hline Max. Wiederfindungsrate [\%] & 100,66 & 90,8 & 98,84 & 105,40 \\
\hline Variationskoeffizient [\%] & 2,51 & 4,78 & 2,18 & 2,36 \\
\hline HEMA & & & & 114,26 \\
\hline Max. Wiederfindungsrate [\%] & 67,42 & 92,18 & 104,18 & 3,19 \\
\hline Variationskoeffizient [\%] & 4,39 & 11,77 & 4,55 & \\
\hline
\end{tabular}

MA: Methacrylat, MMA: Methylmethacrylat; HEMA: 2-Hydroxyethylmethacrylat

Tab. 2: Vergleich der Nachweis- (LOD) und Bestimmungsgrenzen (LOQ) bei manueller und automatisierter Festphasenextraktion (SPE).

\begin{tabular}{llllll} 
& & \multicolumn{2}{c}{ Wasser } & \multicolumn{2}{c}{ Speichel } \\
\hline \multirow{2}{*}{ SPE } & Substanz & LOD $(\mathrm{ppb})$ & LOQ $(\mathrm{ppb})$ & LOD (ppb) & LOQ (ppb) \\
\hline \multirow{2}{*}{ Manuell } & MA & 2,73 & 6,78 & 1,59 & 4,04 \\
\cline { 2 - 6 } & MMA & 11,91 & 30,27 & 5,09 & 12,35 \\
\cline { 2 - 6 } & HEMA & 4,36 & 11,48 & 1,4 & 3,39 \\
\hline \multirow{2}{*}{ Mutomatisiert } & MA & 4,15 & 9,33 & 3,46 & 8,56 \\
\cline { 2 - 6 } & MMA & 16,11 & 41,88 & 9,56 & 24,02 \\
\cline { 2 - 6 } & HEMA & 2,52 & 6,24 & 8,97 & 24,19 \\
\hline
\end{tabular}

MA: Methacrylat; MMA: Methylmethacrylat; HEMA: 2-Hydroxyethylmethacrylat; ppb: parts per billion sich ein ähnliches Bild. Für alle drei Komponenten konnten mittels automatisierter SPE erheblich größere Wiederfindungen von bis $\mathrm{zu}$ 92,96\% (MA), 105,4\% (MMA) sowie $114,26 \%$ (HEMA) im Vergleich zu 56,64\% (MA), 90,8\% (MMA) und 92,18 \% (HEMA) im manuellen Verfahren ermittelt werden.

Die Ergebnisse für die Extraktion aus den Wasserproben zeigen Variationskoeffizienten von 2,51-4,95\% für die manuelle SPE im Vergleich zu Werten von $1,97-4,55 \%$ in der automatisierten SPE. Für die extrahierten Speichelproben sanken die Variationskoeffizienten bei Anwendung der automatisierten SPE deutlich gegenüber dem manuellen Verfahren.

Für die Bestimmung der Nachweisgrenzen (limit of detection, LOD, geringste nachweisbare Analytkonzentration) und Bestimmungsgrenzen (limit of quantification, LOQ, geringste quantifizierbare Analytkonzentration) wurden zehn unabhängige Leerproben am selben Tag prozessiert (Tab. 2).

Auffallend sind hier geringere Werte sowohl für die Nachweis- als auch die Bestimmungsgrenzen für die Extraktion von MMA aus Speichel im Vergleich zur Extraktion aus Wasser.

Die Messpräzision wurde durch zehnfache Messung einer einzelnen Probe ermittelt, die erhaltenen Werte liegen zwischen 0,81 und $3,11 \%$ (Extraktion aus Wasser) bzw. 0,71 und $3,26 \%$ (Extraktion aus Speichel). Für die Ermittlung der Methodenstabilität der automatisierten SPE wurden je zehn Proben extrahiert und in jeweils fünf Teile aufgeteilt. Ein Teil wurde sofort vermessen, während die übrigen Proben eingefroren und an den folgenden vier Tagen aufgetaut und vermessen wurden. Dabei konnten mittlere Variationskoeffizienten von 1,19-4,34\% (Extraktion aus Wasser) bzw. 0,95-2,18\% (Extraktion aus Speichel) ermittelt werden.

\section{Zusammenfassung}

Eine automatisierte Methode der Festphasenextraktion wurde für die Bestimmung von MA, MMA und HEMA in wässrigen Lösungen und künstlichem Speichel entwickelt. Die Ergebnisse zeigen gute Wiederfindungsraten zwischen 92,96 und 114,26\%. Während für die Prozessierung von 24 Proben mittels manueller SPE 187 Minuten erforderlich waren, konnte mit der automatisierten SPE eine Zeitreduktion um 68,98\% auf 58 Minuten erreicht werden. Im Vergleich zur klassischen Flüssig-FlüssigExtraktion hat die SPE einige Vorteile. 
Neben der Zeiteinsparung und damit einer deutlichen Produktivitätserhöhung sind dies vor allem eine erhebliche Reduktion des Lösungsmittelverbrauches sowie eine bessere Probenaufreinigung durch die spezifische Selektivität des verwendeten Sorbensmaterials.

\section{Danksagung}

Die Forschungsarbeiten wurden unterstützt vom Bundesministerium für Bildung und Forschung (FKZ: 03Z1KN11). Die Autoren danken Dr. Steffen Junginger, DI Lars Woinar und Heiko Engelhardt für die konstruktiven Arbeiten. Ferner gilt der Dank Sybille Horn für die Durchführung der experimentellen Aufgaben.

\section{Literatur}

[1] Daun CS (2014) Kontaktallergie auf Dentalmaterialien. Dissertation, Ludwig-Maximilians-Universität München

[2] Leggat PA, Kedjarune U (2003) Toxicity of methyl methacrylate in dentistry. Int Dent J 53:126-131 [3] Schweikl H, Hartmann A, Hiller KA et al. (2007) Inhibition of TEGDMA and HEMA-induced genotoxicity and cell cycle arrest by N-acetylcyteine. Dent Mater 23:688-695 [4] Schweikl H, Spagnuolo G, Schmalz G (2006) Genetic and cellular toxicology of dental resin monomers. J Dent Res 85:870-877

[5] Spahl W, Budzikiewicz H, Geurtsen W (1998) Determination of leachable components from four commercial dental composites by gas and liquid chromatography/ mass spectrometry. J Dent 26:137-145

[6] Rogalewicz R, Batko K, Voelkel A (2006) Identification of organic extractables from commercial resin-modified glass-ionomers using HPLC/MS. J Environ Monit 8:750-758 [7] Fleischer H, Vorberg E, Warkentin M et al. (2014) Qualitative and quantitative determination of methacrylates in dental filling materials. Am Lab 46:10-15

[8] Babidge PJ, Babidge WJ (1994) Determination of methylmalonic acid by high-performance liquid chromatography. Anal Biochem 216:424-426

[9] Garbe C, Reimann H (2005) Dermatologische Rezepturen: Schlüssel zur individualisierten topischen Therapie. Thieme, Stuttgart

[10] Schedler M, Fleischer H, Bandomir J et al. (2018) Evaluation of an Automated Sample Preparation System for Analysis of Methacrylates in Saliva. Proceedings, IEEE International Conference on Automation Science and Engineering (CASE) 2018, München, 1110-1115
[11] Schedler M, Roddelkopf T, Bandomir J et al. (2018) Automated Pre-Sensoric Selectivity System for Determination of Methacrylates from Dental Composite Materials. Proceedings, IEEE International Instrumentation and Measurement Technology Conference (I2MTC) 2018, Houston, 136-140

Funding: Open Access funding provided by Projekt DEAL.

Open Access: Dieser Artikel wird unter der Creative Commons Namensnennun 4.0 International Lizenz veröffentlicht, welche die Nutzung, Vervielfältigung, Bearbeitung, Verbreitung und Wiedergabe in jeglichem Medium und Form erlaubt, sofern Sie den/die ursprünglichen Autor(en) und die Quell

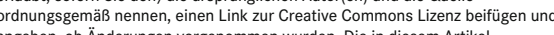
enthaltenen Bilder und sonstiges Drittmaterial unterliegen ebenfalls genannten Creative Commons Lizenz, sofern sich aus der Abbildungslegende nichts anderes ergibt. Sofern das betreffende Material nicht unter der genannten Creative Commons Lizenz steht und die betreffende Handlung nicht nach gesetzlichen Vorschriften erlaubt ist, ist für die oben aufgeführten Weiterverwendungen des Materials die Einwilligung des jeweiligen Rechteinhabers einzuholen. Weitere Details zur Lizenz entnehmen Sie bitte der Lizenzinformation auf http://creativecommons.org/licenses/by/4.0/deed.de.

\section{Korrespondenzadresse:}

Prof. Dr.-Ing. habil. Kerstin Thurow

Center for Life Science Automation (celisca)

Universität Rostock

Friedrich-Barnewitz-Straße 8

D-18119 Rostock

kerstin.thurow@celisca.de

www.celisca.de

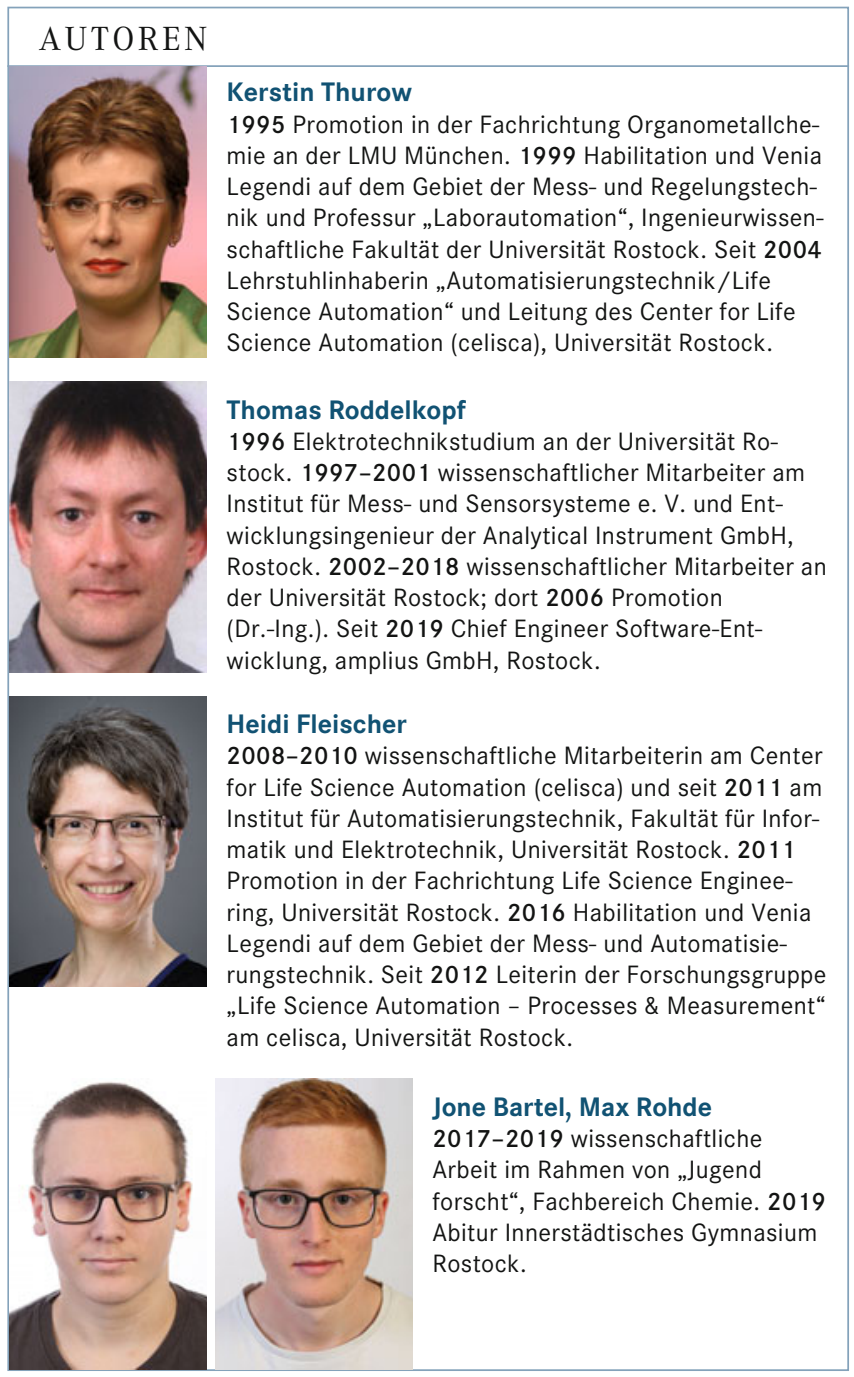

\section{Hier steht eine Anzeige.}

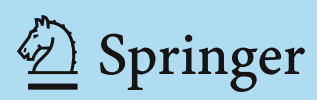

